\title{
Anaesthesia for neuroradiology
}

$M$ ANY recent developments in technology have resulted in great progress in the investigation and treatment of patients with central nervous system diseases by radiological procedures. Anaesthetists may be involved in the care of these patients when the procedures are prolonged or uncomfortable, require complete immobility of the patient, or when the patient does not tolerate the procedure without sedation. The objectives of this lecture will be first, to review the general principles of anaesthesia outside of the operating room as illustrated by the management of patients during magnetic resonance imaging (MRI), and secondly, the discussion of anaesthesia for interventional neuroradiology (INR) with an update of the endovascular treatment of cerebral aneurysms and strokes.

\section{Anaesthesia outside the operating room}

Providing anaesthesia outside of the operating room in an unfamiliar environment requires careful planning and understanding of the patient, the procedure, the available equipment and the hazards of the environment. ${ }^{1}$ The assessment of the patient is the same as for any patient undergoing anaesthesia. The technique of anaesthesia, whether general anaesthesia or conscious sedation, needs to be discussed with the radiologist as well as with the patient. Many procedures can be performed with only sedation and analgesia, whereas general anaesthesia is usually reserved for patients (adults and children) who are uncooperative or where there is another indication. Some of the more difficult procedures in INR such as ablation of an aneurysm with coils, are often performed with general anaesthesia at the request of the neuroradiologist. This is to allow for complete immobility of the patient and the frequent use of apnea for good quality imaging. The usual reason for requesting an anaesthetist in the MRI, is for the uncooperative adult or child, or for the patient who has severe claustrophobia and is unable to lie inside the confined space.

In order to provide adequate anaesthesia, the anaesthetist needs to know what the procedure entails, how it will be performed, and what are the potential
Pirjo H. Manninen MD FRCPC

complications. Good rapport with the radiologist is essential. Preparation of the room and the equipment should be the same as for the anaesthetic care of a patient in the operating room. The standard requirements of monitoring during anaesthesia also apply to these patients, such as the ECG, blood pressure, temperature, $\mathrm{SpO}_{2}$ and $\mathrm{P}_{\mathrm{ET}} \mathrm{CO}_{2}$. Drugs for general anaesthesia, conscious sedation and for emergency resuscitation should be readily available. Emergency equipment for cardiopulmonary resuscitation should be available close by.

When working outside the operating room, the anaesthetist frequently has to fit in amongst all the equipment required for the procedure and often the site is in a remote isolated area. These are difficulties particularly for MRI. There may be limited access to the patient, dark, cold rooms, uncomfortable table for the patient and a hostile environment for electronic monitoring. If an anaesthetist is not frequently present in the radiology suite, then there may be nobody else in room who understands the complexities of anaesthesia. The anaesthetist needs to know where there is assistance available in the event of an emergency, for example a difficult intubation.

The difficulties of providing anaesthesia in the MRI suite are the problems of the magnetic field. ${ }^{2-4}$ The cylindrical magnet surrounds the body, limiting access to the patient. Ferromagnetic objects may be hurled toward the scanner creating a lethal projectile. Large metal objects may interfere with the quality of the image. Many electronic instruments may not function normally close to the magnet. Implantable ferrous magnetic devices such as old cerebrovascular clips, surgical clips and pacemakers are hazardous. Ferrous material is present in most anaesthetic equipment such as ventilators, vaporizers, gas cylinders, laryngoscopes batteries, stethoscopes, connectors and intravenous poles. MRI compatible equipment including anaesthetic machines and monitors are available commercially. Also, one can modify existing equipment. Long tubing is essential for all monitors and intravenous lines. 


\section{Anaesthetic management}

Before the procedure the patient should be assessed thoroughly in preparation for anaesthesia. Routine monitors are used, but if the patient has any medical problems or if specific procedures such as induced hypotension are required, invasive monitoring may be needed. During conscious sedation, oxygen should be administered by nasal prongs or face mask with an adapter to monitor $\mathrm{P}_{\mathrm{ET}} \mathrm{CO}_{2}$. A urinary catheter may be required for prolonged procedures as radiological contrast material acts as an osmotic diuretic. Monitoring of the central nervous system is possible with conscious sedation techniques but, during general anaesthesia, other forms of monitoring may be required, such as EEG, evoked potentials, or transcranial doppler. These techniques are often difficult to perform in the radiology suites due to the hostile electrical environment and lack of space. The post anaesthesia care unit is the ideal place to recover the patient but, frequently, this is far away and one needs to ensure that the patient is stable prior to transport.

\section{Anaesthetic techniques}

When awake neurological assessment is required, conscious sedation is used. Appropriate levels of analgesia and/or sedation allow the patient to be comfortable and immobile, but still responsive at times of assessment. Different techniques and anaesthetic agents have been used to achieve these goals. ${ }^{5-7}$ Various combinations of agents include 1) the traditional "neurolept," fentanyl and droperidol, 2) propofol and opioid, and 3) midazolam and opioid. These drugs may be given as intermittent boluses or as a continuous infusion.

General anaesthesia is used when indicated by the procedure or the patient. There is little information as to the best technique but the same principles of management of patients undergoing surgical procedures apply to these patients.

\section{Interventional neuroradiology}

Interventional neuroradiology involves the invasive endovascular approach to the treatment of vascular lesions in the brain and spine. ${ }^{8,9}$ The goals of INR therapy are 1) definitive, such as the embolization of a dural or spinal fistula, embolization of a spinal or intracerebral AVM, ablation of a cerebral aneurysm by coils, treatment of vasospasm or stenosis by angioplasty, and thrombolytic therapy for embolic stroke, 2) adjunctive therapy involves the preoperative embolization of an AVM or brain tumour, or the test arterial occlusion for an aneurysm or tumour, and 3) palliative treatment such as the intraarterial chemotherapy for malignant brain tumors. ${ }^{10-13}$
The INR procedures are performed by the placement of special catheters mostly via the femoral artery. If the patient is awake the area is infiltrated with a local anaesthetic agent, such as bupivacaine $0.25 \%$. A coaxial catheter is usually inserted up into the carotid or vertebral artery. Through this, various microcatheters can be inserted to deliver drugs and embolic agents to desired locations. The materials used for embolization include a wide variety of different products such as balloons, coils, solid agents consisting of polyvinyl particles, liquid agents such as cyanoacrylates and thrombolytic agents such as urokinase and tissue plasminogen activators. ${ }^{14}$ The actual imaging technology includes high resolution fluoroscopy, and high speed digital subtraction angiography with "road-mapping" functions. A scout film is initially performed and this serves as a mask, which can be subtracted by the computer from all subsequent images in the run so that only the opacified vessels with contrast are visible. The road-mapping is then done by the injection of a bolus of contrast from the proximal coaxial catheter to obtain an image that demonstrates the vascular anatomy. The computer then superimposes this image on light fluoroscopy image so that the radiologist can follow the progress of the radiopaque catheter against the road map. Any movement or motion during this stage of the procedure profoundly degrades the image.

Complications can occur rapidly and can be devastating. ${ }^{15}$ The INR complications fall into two major categories: 1) haemorrhage from a rupture of the lesion or of a vessel, and 2) ischaemia, which can result from thromboembolism, embolic material, vasospasm, hypoperfusion, arterial dissection, stenosis and venous outflow obstruction. When a radiological catastrophe occurs, communication between the anaesthetist and radiologist is vital. Rapid intervention and treatment of the patient is needed. If it is due to haemorrhage with rapid increases in intracranial pressure, the patient may require intubation and hyperventilation with or without induction of anaesthesia, as well as the administration of mannitol. Occasionally, the patient may develop a large clot and require urgent craniotomy in the operating room. If the event is due to ischaemia with a vascular occlusion then deliberate increase in blood pressure may be required, or even the consideration of brain protection. Other complications include allergic reactions to radiographic contrast and the hazard of radiation exposure to the personnel. The amount of radiation exposure decreases in proportion to the square of the distance from the source of radiation. Thus, appropriate shielding should be used and activity near the patient's head should be kept at a minimum. There must be continuous communication to allow for optimal patient care and minimal staff exposure. 


\section{Cerebral aneurysms}

Major strides have been made in the management of patients with cerebral aneurysms by endovascular techniques. ${ }^{12}$ Initially, detachable balloons were used but now the most common technique is with electrically detachable platinum coils. ${ }^{16}$ This has been shown to be effective because the flow into the aneurysm is disrupted by mechanical packing, with subsequent thrombosis and clotting. Endovascular treatment of aneurysms offers advantages, including avoidance of a craniotomy and surgical manipulation and, at times, general anaesthesia. The preference for general anaesthesia or for conscious sedation varies among institutions. With either technique of anaesthesia, these patients, especially if they have had a recent bleed, present a challenge to the anaesthetist. The same considerations as in the operating room, such as control of systemic blood pressure, aneurysmal transmural pressure and cerebral blood flow are needed. Maintenance of anaesthesia may be difficult as there is no surgical stimulation.

\section{Stroke}

The neurological deficits caused by the ischaemic events of a thromboembolic stroke can be reversed by intraarterial thrombolytic therapy with injection of tissue plasminogen activator or urokinase resulting in the recanalization of the artery. ${ }^{13,17}$ This must be performed within six hours from the ischaemic event. The major risk of this procedure is haemorrhage. These patients will require either general anaesthesia or conscious sedation depending on their clinical status. The procedures are usually emergency cases performed at any hour of the day or night. An important consideration is control of systemic blood pressure. Hypotension will worsen the ischaemic event and hypertension may result in haemorrhage especially if a hyperperfusion injury occurs after thrombolysis of the clot.

\section{Conclusion}

The field of radiology is a rapidly expanding and growing area in the investigation and treatment of central nervous system diseases. The role of the anaesthetist will continue to be important in the management of these patients. The anaesthetist must be prepared to modify and adapt to the changes in techniques, procedures, and to the requirements of the neuroradiologist and patient.

\section{References \\ 1 Hughes $C W$. Anesthesia outside of the operating room. Seminars in Anesthesia 1990; 1X: 190-6. \\ 2 Menon DK, Peden CJ, Hall AS, Sargentoni J, Whitwam $J G$. Magnetic resonance for the anaesthetist. Part I:}

physical principles, applications, safety aspects. Anaesthesia 1992; 47: 240-55.

3 Peden CJ, Menon DK, Hall AS, Sargentoni J, Whitwam $J G$. Magnetic resonance for the anaesthetist. Part II: anaesthesia and monitoring in MR units. Anaesthesia 1992; 47, 508-17.

4 Patteson SK, Chesney JT. Anesthetic management for magnetic resonance imaging: problems and solutions. Anesth Analg 1992; 74: 121-8.

5 O'Mahony BJ, Bolsin SNC. Anaesthesia for closed embolisation of cerebral arteriovenous malformations. Anaesth Intensive Care 1988; 16: 318-23.

6 Manninen PH, Gignac EM, Gelb AW, Lownie SP. Anesthesia for interventional neuroradiology. J Clin Anesth 1995; 7: 448-52.

7 Manninen PH, Chan ASH, Papworth D. Conscious sedation for interventional neuroradiology: a comparison of midazolam and propofol infusion. Can J Anaesth 1997; 44: 26-30.

8 Eskridge JM. Interventional neuroradiology. Radiology 1989; 172: 991-1006.

9 Young WL, Pile-Spellman J. Anesthetic considerations for interventional neuroradiology. Anesthesiology 1994; 80: 427-56.

10 Debrun $G$, Vinuela F, Fox $A$, Drake CG. Embolization of cerebral arteriovenous malformations with bucrylate. J Neurosurg 1982; 56: 615-27.

11 Nelson PK, Setton A, Choi IS, Ransohoff J, Berenstein A. Current status of interventional neuroradiology in the management of meningiomas. Neurosurg Clin $\mathrm{N}$ Am 1994; 5: 235-59.

12 Higashida RT, Halbach VV, Dowd CF, Hieshima GB. Intracranial aneurysms. Evolution and future role of endovascular techniques. Neurosurg Clin N Am 1994; 5: 413-25.

13 del Zoppo GJ, Ferbert A, Otis $S$, et al. Local intra-arterial fibrinolytic therapy in acute carotid territory stroke. A pilot study. Stroke 1988; 19: 307-13.

14 Khayata $M H$, Dean BL, Spetzler RF. Materials and embolic agents for endovascular treatment. Neurosurg Clin N Am 1994; 5: 475-84.

15 Lownie SP. Clinical and technical complications of endovascular therapy in the central nervous system. Seminars in Interventional Radiology 1993; 10: 243-53.

16 Guglielmi G, Viuela F. Intracranial aneurysms. Guglielmi electrothrombotic coils. Neurosurg Clin $\mathbf{N}$ Am 1994; 5: 427-35.

17 Wardlaw JM, Warlow CP. Thrombolysis in acute ischemic stroke: does it work? Stroke 1992; 23: 1826-39. 


\section{L'anesthésie pour la neuroradiologie}

$\mathrm{P}$ LUSIEURS des développements technologiques récents ont fait considérablement progresser les domaines de l'investigation et du traitement radiologiques des maladies nerveuses. Les anesthésistes sont requis quand les interventions sont prolongées ou inconfortables, quand le patient doit demeurer complètement immobile ou quand il ne peut tolérer l'intervention sans sédation. Les objectifs de cette communication seront d'abord de faire un survol des principes généraux de l'anesthésie à l'extérieur des salles d'opération comme l'imagerie pas résonance magnétique (IRM) et, deuxièmement, de discuter l'anesthésie pour les interventions neuroradiologiques avec une mise à jour sur le traitement endovasculaire de l'anévrisme et des accidents cérébrovasculaires (ACV).

L'anesthésie à l'extérieur des salles d'opération L'administration de l'anesthésie à l'extérieur de la salle d'opération, dans un environnement avec lequel il n'est pas familier, exige de l'anesthésiste une planification minutieuse et la connaissance du patient, de l'intervention, du matériel disponible et des désavantages de cet environnement. ${ }^{1}$ L'évaluation du patient est la même que celle de tout autre patient programmé pour l'anesthésie générale. La technique de l'anesthésie, que ce soit l'anesthésie générale ou une sédation légère, doit être discutée avec le patient de même qu'avec le radiologiste. Plusieurs interventions peuvent être effectuées seulement avec de la sédation et de l'analgésie, tandis que l'anesthésie générale est réservée aux patients (enfants ou adultes peu coopératifs) ou pour d'autres indications. Quelques-unes des interventions neuroradiologiques plus difficiles comme l'ablation d'un anévrisme avec des spirales sont souvent réalisées sous anesthésie générale à la demande du radiologiste. Ceci permet d'obtenir l'immobilité complète du patient et de provoquer de l'apnée au besoin pour obtenir une image de bonne qualité. La présence de l'anesthésiste pour l'IRM est requise ordinairement lorsqu'un enfant ou un adulte refuse de collaborer ou lorsqu'un patient souffre d'une claustrophobie qui le rend incapable de s'allonger dans un espace confiné.

Pour procurer une anesthésie satisfaisante l'anesthésiste doit connaître en quoi consiste l'intervention, de quelle façon elle se déroule et quelles sont ses complica- tions potentielles. Il est essentiel de bien s'entendre avec le radiologiste. La salle et le matériel sont préparés de la même façon qu'au bloc opératoire. Les normes de monitorage usuelles comme l'ECG, la pression artérielle, la température, la $\mathrm{SpO}_{2}$ et la $\mathrm{P}_{\mathrm{ET}} \mathrm{CO}_{2}$ s'appliquent à ces patients. Les agents nécessaires pour l'anesthésie générale, la sédation vigile et la réanimation doivent être à portée de main. L'équipement d'urgence pour la réanimation cardio-pulmonaire doit être rapidement disponible.

Souvent, lorsqu'il travaille à l'extérieur de la salle d'opération, en plus de se trouver dans un secteur isolé, l'anesthésiste doit se blottir au milieu de l'appareillage requis pour l'intervention. C'est particulièrement vrai avec l'IRM. Ordinairement, l'accès au patient est limité, la salle est froide et sombre, la table d'examen inconfortable et l'environnement se prête mal au monitorage électronique. Lorsque l'anesthésiste ne fréquente que rarement les salles de radiologie, il se produit que personne dans la salle ne comprend la complexité de l'anesthésie. L'anesthésiste doit savoir où trouver de l'aide en cas d'urgence, ex. pour une intubation difficile.

Dans la salle d'IRM, le champ magnétique est au coeur des problèmes de l'anesthésiste. ${ }^{2-4}$ L'aimant cylindrique enveloppe le corps et limite l'accès au patient. Les objets ferromagnétiques peuvent se transformer en projectiles et être aspirés par l'aimant. Les objets de métal volumineux peuvent nuire à la qualité de l'image. Plusieurs des instruments électroniques fonctionnent mal à proximité de l'aimant. Les instruments métalliques implantés comme les clips cérébrovasculaires, les clips hémostatiques chirurgicaux et les pacemakers sont menacés. Le fer est présent dans la plupart des appareils en usage en anesthésie comme les ventilateurs, évaporateurs, bonbonnes, piles de laryngoscope, stéthoscopes, raccords et tiges de solutés. Nous disposons maintenant d'appareils compatibles avec l'IRM dont des appareils d'anesthésie et des moniteurs. On peut aussi modifier l'équipement actuel. Il est essentiel d'utiliser des câbles et des tubulures pour solutés de d'une longueur appropriée.

\section{Conduite de l'anesthésie}

Avant l'intervention, le patient subira une évaluation complète en fonction de l'anesthésie. On utilisera les moniteurs habituels mais si la malade présente des prob- 
lèmes médicaux particuliers et si des techniques spéciales comme l'hypotension contrôlée sont requises, on aura recours au monitorage invasif. Pendant une sédation vigile, de l'oxygène devrait être administré par tubulure à éperons nasaux ou par masque avec un adaptateur pour la mesure de la $\mathrm{P}_{\mathrm{ET}} \mathrm{CO}_{2}$. Comme le matériel de contraste a les propriétés d'un diurétique osmotique, il est nécessaire d'insérer une sonde urinaire pour les interventions de longue durée. Il est possible de monitorer le système nerveux central sous sédation vigile, mais sous anesthésie générale, il faut parfois recourir à d'autres formes de monitorage telles que l'ÉEG, les potentiels évoqués ou le Doppler transcrânien. Ces techniques sont souvent difficiles à réaliser dans un département de radiologie à cause d'un environnement électrique mal adapté et du manque d'espace. La salle des soins postanesthésiques demeure l'endroit idéal pour récupérer, mais souvent, elle se trouve à une grande distance et on doit s'assurer que le patient est stable avant d'entreprendre son transfert.

\section{Les techniques anesthésiques}

Chez le sujet conscient, la sédation vigile permet l'évaluation de l'état neurologique. Des niveaux appropriés d'analgésie et de sédation assurent confort et immobilité, mais le patient doit être capable de répondre au moment approprié. Différentes techniques et agents anesthésiques permettent d'atteindre cet objectif. $^{5-7}$ Les associations d'agents les plus efficaces sont: 1) le fentanyl et le dropéridol pour une neuroleptanalgésie traditionnelle, 2) le propofol et un morphinique, et 3) le midazolam et un morphinique. Ils s'administrent en bolus ou en perfusion.

Le patient et l'intervention déterminent l'indication de l'anesthésie générale. Pour ce qui est du choix de la technique, les mêmes principes qu'en salle d'opération s'appliquent à ces patients.

\section{Les interventions neuroradiologiques}

En neuroradiologie, les interventions pour le traitement des lésions cérébrovasculaires et de la moelle épinière comportent une approche endovasculaire. ${ }^{8,9}$ Les objectifs de ces interventions sont: 1) le traitement définitif, ex. l'embolisation d'une fistule dure-mérienne ou rachidienne, l'embolisation d'une malformation artérioveineuse (MAV) rachidienne ou intracérébrale, l'ablation d'un anévrisme cérébral avec des spirales, le traitement d'un vasospasme ou d'une sténose par angioplastie, la thérapie thrombolytique pour l'ACV embolique, 2) le traitement d'appoint par l'embolisation préopératoire d'une MAV ou d'une tumeur cérébrale, ou pour vérifier l'occlusion artérielle d'un anévrisme ou d'une tumeur, et 3) le traite- ment palliatif comme la chimiothérapie intra-artérielle pour une tumeur cérébrale maligne. ${ }^{10-13}$

Les interventions neuroradiologiques sont la plupart du temps effectuées par l'insertion d'un cathéter par l'artère fémorale. Si le patient est éveillé, la région est infiltrée avec un anesthésique local comme la bupivacaïne $0,25 \%$. Un cathéter coaxial est ordinairement introduit jusqu'à la carotide ou l'artère vertébrale. Par ce cathéter, on introduit différents microcathéters pour délivrer des médicaments ou du matériel embolisant aux endroits désirés. Pour l'embolisation, on utilise plusieurs matériaux et produits comme des ballonnets, des spirales, des agents solides constitués de particules d'alcool de polyvinyle, des produits liquides comme les cyanoacrylates et des thrombolytiques comme l'urokinase et les activateurs du plasminogène tissulaire. ${ }^{14} \mathrm{La}$ technologie d'imagerie en usage comprend la fluoroscopie à haute résolution et l'angiographie de soustraction numérisée à haute vitesse avec fonction cartographique (road-mapping). On réalise d'abord un film guide qui sert de masque qui sera soustrait par l'ordinateur de toutes les images subséquentes de la série de sorte que seuls les vaisseaux opacifiés par le contraste seront visibles. La cartographie est alors exécutée par l'injection d'un bolus de matériel de contraste à partir du cathéter coaxial proximal pour obtenir une image qui révèle l'anatomie vasculaire. L'ordinateur superpose ensuite cette image sur une image de fluoroscopie lumineuse de sorte que la radiologiste peut suivre le progrès du cathéter sur la «carte routière».Tout mouvement pendant ce stage de l'intervention altére l'image profondément.

En neuroradiologie, les complications surviennent rapidement et sont catastrophiques ${ }^{15}$ Elles appartiennent à deux catégories principales : 1) l'hémorragie par rupture d'une lésion ou d'un vaisseau, et 2) l'ischémie, par thromboembolie, embolie solide, vasospasme, hypoperfusion, dissection artérielle, sténose et obstruction du retour veineux. Quand un désastre radiologique survient, la communication entre anesthésiste et radiologiste est vitale. Il faut intervenir et traiter immédiatement l'hémorragie qui a pour effet d'augmenter rapidement la pression intracrânienne; dans ce cas, il faut intuber et hyperventiler avec ou sans induction de l'anesthésie, et administrer du mannitol. Occasionnellement, l'expansion d'un caillot nécessitera une craniotomic d'urgence en salle d'opération. En cas d'ischémie par occlusion vasculaire, le traitement consiste à augmenter la pression artérielle ou à mettre en marche des mesures de protection cérébrale. Une réaction allergique au médium de contraste est toujours possible. L'exposition à la source de radiation constitue un danger pour le personnel. Il faut utiliser un blindage protecteur et réduire au minimum toutes les manoeu- 
vres autour de la tête du patient. Pour le patient, les bonnes communications permettent une prise en charge optimale, et pour le personnel, une réduction au minimum de l'exposition aux radiations.

\section{L'anéprisme cérébral}

Le traitement de l'anévrisme cérébral par les techniques endovasculaires a fait beaucoup de progrès. ${ }^{12} \mathrm{Au}$ début, on se servait des ballons détachables mais maintenant la technique courante utilise des spirales de platine qui se détachent électriquement. ${ }^{16}$ L'efficacité de cette technique vient de ce que le débit intra-anévrismal est interrompu par tassement mécanique avec thrombose et coagulation subséquentes. Le traitement endovasculaire de l'anévrisme est avantageux parce qu'elle permet d'éviter la craniotomie et les manipulations chirurgicales et parfois l'anesthésie générale. Le choix entre anesthésie générale et sédation vigile varie selon les institutions. Quelle que soit la technique d'anesthésie, ces patients présentent tout un défi pour l'anesthésiste, surtout lorsqu'ils ont saigné récemment. Comme en salle d'opération, il faut contrôler la pression artérielle systémique, la pression transmurale anévrismale et débit sanguin cérébral. L'absence de stimulation chirurgicale ne facilite pas le maintien de l'anesthésie.

\section{L'ACV}

Le déficit neurologique causé par la séquence ischémique de l'ACV thromboembolique peut être renversé par la thérapie intra-artérielle thrombolytique qui consiste à injecter de l'activateur du plasminogène tissulaire ou de l'urokinase dans le but de recanaliser l'artère. ${ }^{13,17} \mathrm{Ceci}$ doit être fait dans les six heures qui suivent l'accident ischémique. L'hémorragie constitue le risque principal de cette intervention. Dépendant de leur état, ces patients auront besoin soit d'une anesthésie générale soit d'un sédation vigile. Ces interventions se font toujours en urgence, indépendamment de l'heure, que ce soit le jour ou la nuit. Il est important de garder le contrôle sur la pression artérielle systémique. L'hypotension tend à aggraver l'accident ischémique tandis que l'hypertension peut provoquer une hémorragie et, particulièrement, une lésion par hyperperfusion, après la thrombolyse du caillot.

\section{Conclusion}

Le domaine de la radiologie est en pleine expansion et occupe une place croissante dans l'investigation et le traitement des maladies du système nerveux central. L'anesthésiste continuera de jouer un rôle important dans la prise en charge de ces patients. L'anesthésiste doit être prêt à modifier et à adapter ses techniques aux nouvelles interventions et aux besoins du neuroradiologiste de même qu'à ceux du patient.

\section{Références}

(Voir page R36) 